\title{
Statin-Induced Immune-Mediated Necrotizing Myopathy: An Increasingly Recognized Inflammatory Myopathy
}

\author{
Oana P. Stroie ${ }^{1}$, Joshua Boster ${ }^{2}$, Luke Surry ${ }^{3}$
}

1. Internal Medicine, Uniformed Services University of the Health Sciences, Bethesda, USA 2. Internal Medicine, Brooke Army Medical Center, Fort Sam Houston, San Antonio, USA 3. Internal Medicine, San Antonio Uniformed Services Health Education Consortium, San Antonio, USA

Corresponding author: Luke Surry, luke.t.surry.mil@mail.mil

\begin{abstract}
Statin-induced immune-mediated necrotizing myopathy, also known as anti-3-hydroxy-3-methylglutarylcoenzyme A reductase (anti-HMGCR) myopathy, is an inflammatory myopathy that is triggered by statin exposure and persists after statin discontinuation. It is a rare side effect of statins, distinct from the more commonly recognized statin-induced myalgia, that is challenging to diagnose and treat. We describe a case of anti-HMGCR myopathy in a 59-year-old male with a prior history of statin intolerance presenting with markedly elevated creatinine kinase, myoglobinuria, and one month of progressive proximal muscle weakness after restarting atorvastatin 10 months prior to admission. High-dose glucocorticoids led to rapid clinical improvement, although the patient relapsed upon tapering. Remission was attained at three months after combination therapy with azathioprine, intravenous immunoglobulin, and a prolonged prednisone taper.
\end{abstract}

Categories: Internal Medicine, Allergy/Immunology, Rheumatology

Keywords: necrotizing myopathy, myositis, immune-mediated, anti-hmgcr

\section{Introduction}

Statin drugs, ubiquitous in middle and advanced age for the management of atherosclerosis, are increasingly associated with autoimmune disease. Statins are generally considered to be safe and effective with a benign side effect profile, although myalgia is a common complaint. Statin-induced myalgias are due, in part, to the inhibition of cholesterol biosynthetic pathways that may, in turn, lead to upregulation of cholesterol biosynthesis-related genes that subsequently results in reduced skeletal muscle size, protein synthesis, and muscle atrophy [1]. This phenomenon is generally self-limited. Alternatively, inflammatory myopathies are caused by autoimmune-mediated damage to muscle tissue leading to proximal muscle weakness. Among the most well-known inflammatory myopathies are polymyositis, dermatomyositis, and inclusion body myositis. The presence of extramuscular involvement, autoantibodies, and histological examination are used to differentiate between the different subtypes [2]. However, there is a rising incidence of a novel subset of statin-induced immune-mediated necrotizing myopathy (IMNM) that is distinct from statin-induced

Review began $04 / 26 / 2020$ Review began $04 / 26 / 2020$
Review ended 04/28/2020 Published 05/05/2020

\section{(c) Copyright 2020}

Stroie et al. This is an open access article distributed under the terms of the Creative Commons Attribution License CC-BY 4.0., which permits unrestricted use, distribution, and reproduction in any medium, provided the original author and source are credited. myalgias and previously described inflammatory myopathies. IMNM is rare with an estimated incidence of approximately two to three of every 100,000 patients treated with statins [3].

IMNM is an increasingly recognized form of inflammatory myopathy. There are two subtypes that are distinguished based on specific autoantibodies: anti-3-hydroxy-3-methylglutaryl-coenzyme A reductase (anti-HMGCR) and anti-signal recognition particle (SRP). Patients with anti-HMGCR myopathy generally have a history of statin use and are typically older than patients with anti-SRP myopathy [4].

In anti-HMGCR myopathy, the immune system becomes sensitized to increased levels of HMG-CoA reductase that is upregulated by statins, which results in a necrotizing immune-mediated injury to cells that express this enzyme [5]. These autoantibodies continue to persist long after statin discontinuation and result in ongoing tissue damage. Similar to other inflammatory myopathies, statin-induced anti-HMGCR myopathy is distinguished by a specific antibody assay. To our knowledge, there are no controlled trials that have compared the different therapeutic regimens utilized for anti-HMGCR myopathy, but the best evidence is for immunosuppression. This case illustrates the diagnostic pitfalls and response to treatment for this rare condition.

\section{Case Presentation}

A 59-year-old Caucasian man with a history of hypertension, hyperlipidemia, and type 2 diabetes mellitus presented to emergency department after preprocedural labs, for a scheduled liver biopsy to further evaluate persistently elevated liver-associated enzymes (LAEs), revealed a significantly elevated creatine kinase (CK) concerning for rhabdomyolysis. He complained of extreme fatigue and muscle soreness for the past month with difficulty climbing stairs and raising his arms. He denied any preceding fever, chills, rashes, joint pain, 
dysphagia, diplopia, or any recent systemic infections. He had no history of autoimmune disease, malignancy, or hereditary disease. Ten months prior to presentation, the patient restarted atorvastatin 40 mg, following a one-year discontinuation secondary to elevated LAEs.

Physical exam findings were notable for reduced muscle strength symmetrically with hip flexion $2 / 5$, knee flexion 4/5, shoulder abduction 4/5, and elbow flexion $4 / 5$. Gait was stable and he was able to walk unassisted. There was $1+$ pitting edema in the bilateral lower extremities. No fasciculation, atrophy, or swelling of the affected muscles was noted. No skin rashes. Heart, lung, and abdominal examinations revealed no abnormalities and vital signs were within normal ranges.

Laboratory evaluation was notable for significantly elevated CK 17,145 IU/L (N: 24-170 IU/L), elevated aspartate transaminase $449 \mathrm{U} / \mathrm{L}(\mathrm{N}: 5-40 \mathrm{U} / \mathrm{L})$, alanine transaminase $397 \mathrm{U} / \mathrm{L}(\mathrm{N}: 4-41 \mathrm{U} / \mathrm{L})$, and normal alkaline phosphatase. Urinalysis revealed 78 red blood cells and myoglobinuria $993 \mathrm{ng} / \mathrm{mL}(\mathrm{N}: 0-13 \mathrm{ng} / \mathrm{mL})$. Renal function panel, thyroid panel, and complete blood count were within normal limits. Urine drug screen, antinuclear antibody, and myositis panels were negative.

The patient was admitted to the general medical floor with the initial diagnosis of statin induced rhabdomyolysis based on an elevated CK and myoglobinuria. Atorvastatin was discontinued, and aggressive intravenous (IV) hydration was initiated. The patient initially improved with a precipitous drop in CK after the first 24 hours. Beyond the first day, CK elevation persisted despite ongoing aggressive IV hydration. On hospital day 6, IMNM was considered, and the anti-HMGCR antibody test and MRI of thighs were obtained for further evaluation. MRI of the thighs revealed diffuse myositis throughout hamstrings, quadriceps, abductor, and gluteal muscles with trace surrounding soft tissue edema (Figure 1). Anti-HMGCR assay results eventually revealed strongly positive anti-HMGCR antibodies at >200 (N: 0-20) after hospital discharge. 


\section{Cureus}
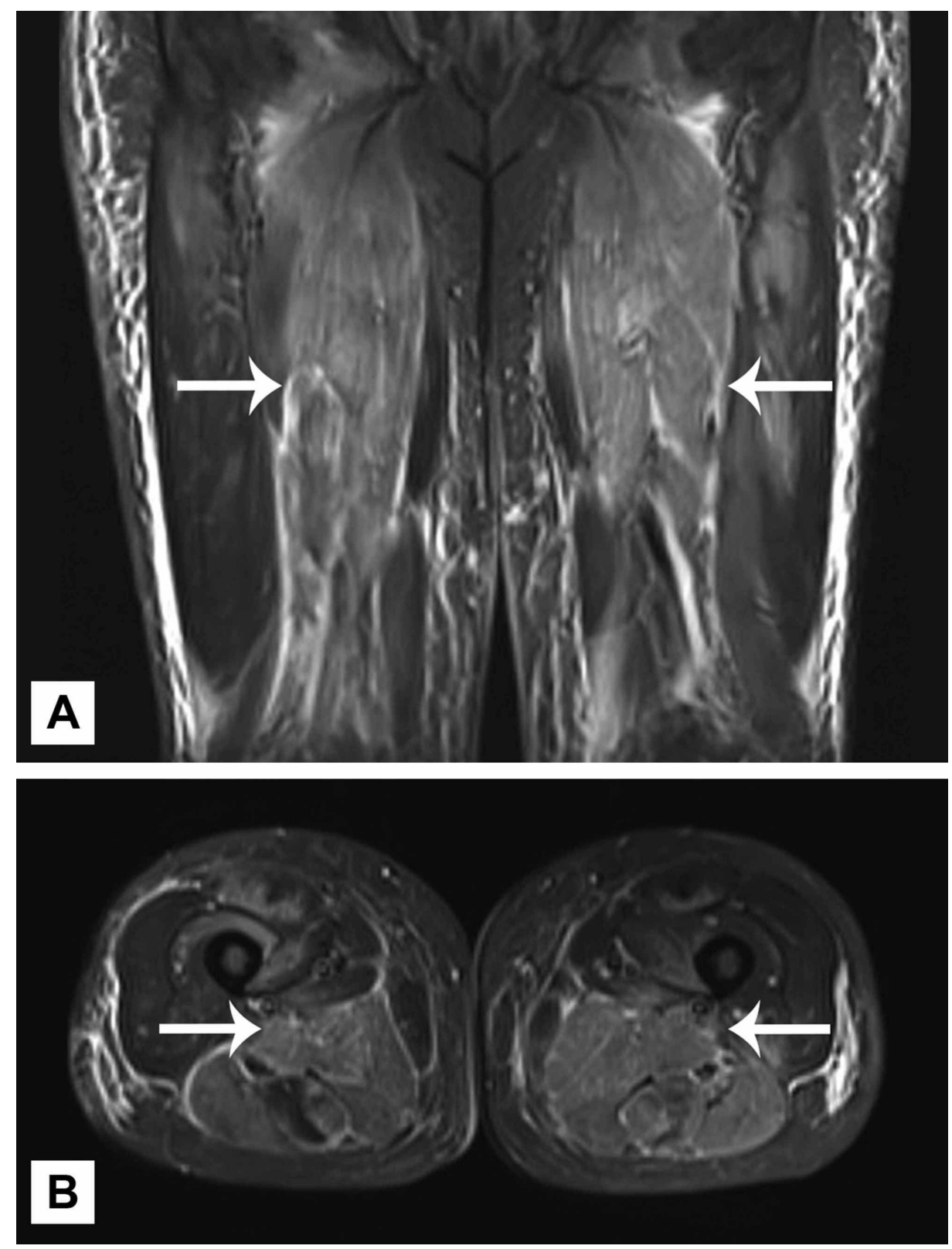

FIGURE 1: Coronal (A) and axial (B) STIR MRI sequences showing diffuse myositis.

STIR, short-TI inversion recovery.

Based on the MRI evidence of diffuse myositis, failure to improve despite aggressive hydration, and an otherwise negative serological evaluation, the patient was started empirically on $80 \mathrm{mg}$ of prednisone for suspected statin-induced IMNM. He demonstrated a rapid response to glucocorticoid therapy and was discharged home with plan to continue prednisone $80 \mathrm{mg}$ by mouth daily with slow taper over the following month.

Prednisone therapy led to a significant improvement in symptoms; however, initial tapering to $40 \mathrm{mg}$ resulted in worsening symptoms and a readmission to the medical floor. Combination therapy was initiated with prednisone $80 \mathrm{mg}$ daily, azathioprine, and intravenous immunoglobulin (IVIG). IVIG infusions were administered every four weeks and azathioprine was titrated up to $200 \mathrm{mg}$ daily coinciding with a slow prednisone taper. The patient responded well, with gradual symptomatic resolution and improvement in muscle strength with normalization of his CK occurring over several months (Figure 2). 


\section{Cureus}

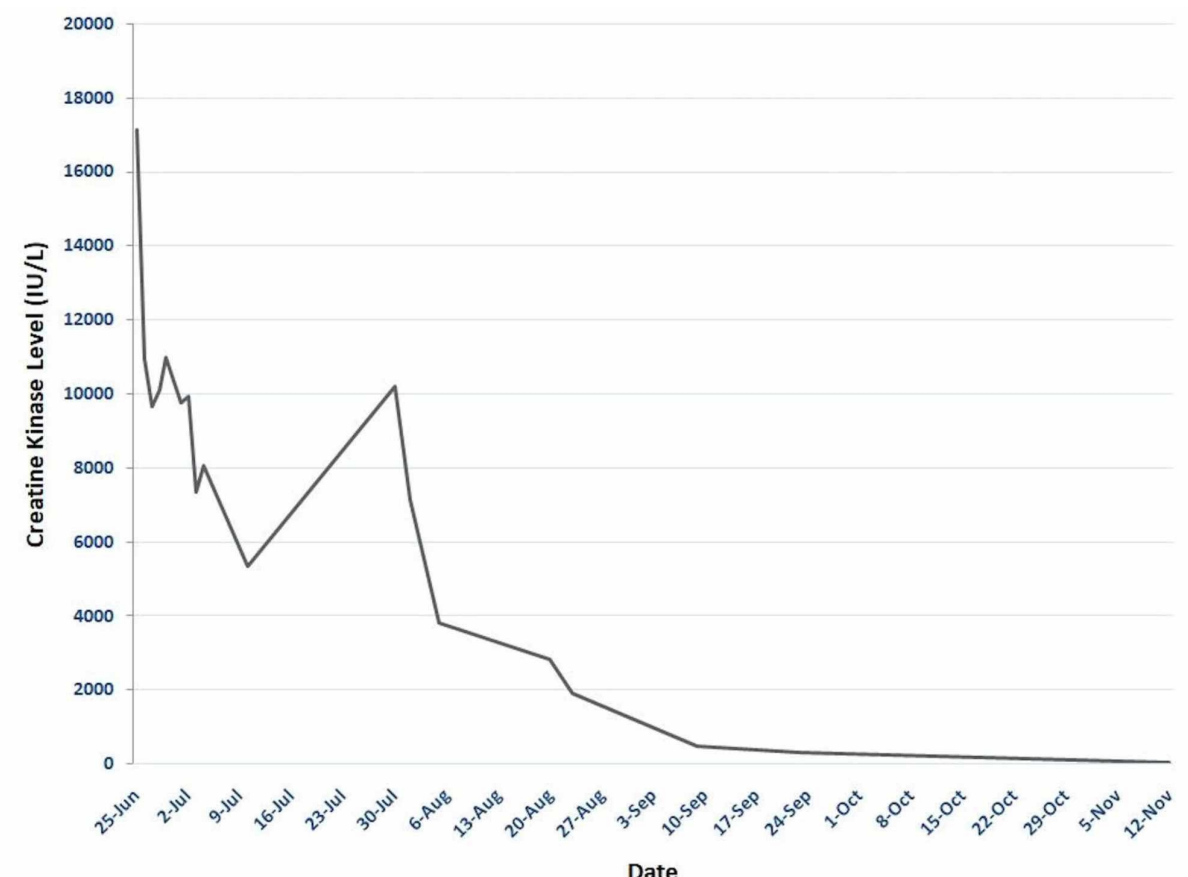

Date

FIGURE 2: Creatine kinase levels and response to therapy over time.

Jun, June; Jul, July; Aug, August; Sep, September; Oct, October; Nov, November.

Discussion

Inflammatory myopathies are a family of rare autoimmune diseases that often pose a diagnostic challenge due to their relative unfamiliarity and broad differential of symptom presentation. The ubiquitous use of statins for the treatment of cardiovascular disease has unmasked a new subset of inflammatory myopathies, anti-HMGCR myopathy that is associated with statin exposure. Failed response to initial treatment in patients presenting with proximal muscle weakness and history of statin use should prompt consideration of alternative diagnoses to include inflammatory myopathies. Unlike other inflammatory myopathies, antiHMGCR myopathy has not been found to be significantly associated with malignancy. It can manifest at any time in people with current or past history of statin use, and in some cases, in the absence of statin use secondary to exposure to statin-like compounds found in foods and dietary supplements like oyster mushrooms and red yeast rice [6].

Inflammatory myopathies are characterized by progressive proximal muscle weakness and markedly elevated CK. The inflammatory etiology in dermatomyositis, polymyositis, and inclusion body myositis is due to a Tcell-mediated immune response against muscle fibers that can be identified on muscle biopsy. In contrast, IMNMs are due to an antibody-mediated immune reaction against muscle fibers that can be identified on biopsy by the presence of macrophages and necrotic fibers undergoing phagocytosis [1,4]. Muscle biopsy is not necessary for the diagnosis as it is less specific for IMNMs than immunoassays [4].

The mainstay of treatment for statin-induced IMNM is immunosuppression. Anti-HMGCR myopathy is difficult to treat due to continued antibody production long after discontinuation of the offending agent [4]. There are no established guidelines for the management of anti-HMGCR myopathy in regard to both optimal treatment regimen and duration. Our patient was started on a high dose of prednisone with a prompt response to therapy followed by a relapse upon initial tapering. Increasingly, evidence suggests combination therapy with high-dose glucocorticoids, methotrexate, azathioprine, or IVIG to be more efficacious in sustaining remission [7]. In patients with diabetes and other cardiovascular comorbidities, a corticosteroid free induction and maintenance may be preferred to minimize the risk of steroid complications [8]. In some cases, even monotherapy with IVIG may be considered [9].

Our patient sustained complete remission within the first three months after initiation of combination therapy with prednisone, IVIG, and azathioprine. This case demonstrates the importance of following serum CK levels closely to monitor response to therapy prior to glucocorticoid tapering.

\section{Conclusions}

Anti-HMGCR myopathy is an IMNM that is associated with statin use. This diagnosis remains relatively rare, 
and it presents with several diagnostic and therapeutic challenges. Optimal management is unclear, but aggressive immunosuppression is effective, although early glucocorticoid tapering in our patient was associated with a recurrence of symptoms. Further research is needed to identify optimal treatment regimens and duration of therapy for these patients.

\section{Additional Information \\ Disclosures}

Human subjects: Consent was obtained by all participants in this study. Brooke Army Medical Center Institutional Review Board issued approval NA. Approved for publication and dissemination by the public affairs office. Conflicts of interest: In compliance with the ICMJE uniform disclosure form, all authors declare the following: Payment/services info: All authors have declared that no financial support was received from any organization for the submitted work. Financial relationships: All authors have declared that they have no financial relationships at present or within the previous three years with any organizations that might have an interest in the submitted work. Other relationships: All authors have declared that there are no other relationships or activities that could appear to have influenced the submitted work.

\section{Acknowledgements}

The views expressed are solely those of the authors and do not reflect the official policy or position of the Uniformed Services University, US Army, US Navy, US Air Force, Department of Defense, or the US Government.

\section{References}

1. Morikawa S, Murakami T, Yamazaki H, et al.: Analysis of the global RNA expression profiles of skeletal muscle cells treated with statins. J Atheroscler Thromb. 2005, 12:121-131. 10.5551/jat.12.121

2. Selva-O'Callaghan A, Pinal-Fernandez I, Trallero-Araguas E, Milisenda JC, Grau-Junyent JM, Mammen AL: Classification and management of adult inflammatory myopathies. Lancet Neurol. 2018, 17:816-828. 10.1016/s1474-4422(18)30254-0

3. Mammen AL: Statin-associated autoimmune myopathy. N Engl J Med. 2016, 374:664-669. 10.1056/NEJMra1515161

4. Ge Y, Lu X, Peng Q, Shu X, Wang G: Clinical characteristics of anti-3-hydroxy-3-methylglutaryl coenzyme A reductase antibodies in Chinese patients with idiopathic inflammatory myopathies. PLoS One. 2015, 10:e0141616. 10.1371/journal.pone.0141616

5. Mammen AL, Chung T, Christopher-Stine L, et al.: Autoantibodies against 3-hydroxy-3-methylglutarylcoenzyme A reductase in patients with statin-associated autoimmune myopathy. Arthritis Rheum. 2011, 63:713-721. 10.1002/art.30156

6. Tiniakou E, Pinal-Fernandez I, Lloyd TE, et al.: More severe disease and slower recovery in younger patients with anti-3-hydroxy-3-methylglutaryl-coenzyme A reductase-associated autoimmune myopathy. Rheumatology. 2017, 56:787-794. 10.1093/rheumatology/kew470

7. Schmidt J: Current classification and management of inflammatory myopathies . J Neuromuscul Dis. 2018, 5:109-129. 10.3233/jnd-180308

8. Meyer A, Troyanov Y, Drouin J, et al.: Statin-induced anti-HMGCR myopathy: successful therapeutic strategies for corticosteroid-free remission in 55 patients. Arthritis Res Ther. 2020, 22:5. 10.1186/s13075 019-2093-6

9. Mammen AL, Tiniakou E: Intravenous immune globulin for statin-triggered autoimmune myopathy . N Engl J Med. 2015, 373:1680-1682. 10.1056/NEJMc1506163 